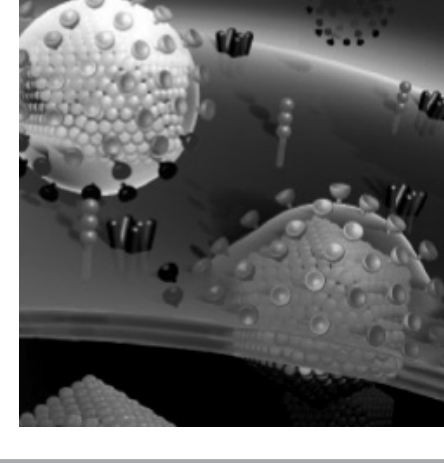

\title{
Management of late presentation in HIV: what the data tell us
}

\author{
"The most effective strategy would probably be directed to the education of relevant \\ healthcare providers coordinated with an increased public awareness and drive towards \\ reducing stigma through national information campaigns."
}

The recognition of more than 10 years ago that effective combination antiretroviral therapy (ART) could decrease morbidity and mortality from HIV infection led to an initial optimism that AIDS would become a thing of the past [1]. In some respects (and in some parts of the world), this could be potential realized; appropriately treated patients are living longer and the risk of progression to AIDS or death is much reduced with effective ART. The emphasis has shifted within treated cohorts away from AIDS illnesses, towards managing and reducing morbidity and mortality from non-AIDS illnesses such as liver disease, cardiovascular disease and malignancy [2].

However, patients - even those living in regions with ready access to ART - are still presenting with AIDS-defining illnesses, many of whom remain undiagnosed until they present with AIDS as the first clinical manifestation of their HIV infection late in their disease. These 'late presenters' are a significant ongoing challenge to the effective management of HIV infection and underscore the need to continually investigate and understand the optimum management of symptomatic HIV infection in its latter stages.

Rates of late presentation differ between cohorts depending on the criterial for defining late presentation (such as advanced clinical staging by the WHO or CDC classification, presentation with an AIDS-defining illness or presentation with $\mathrm{CD} 4{ }^{+} \mathrm{T}$-cell count $<200$ or $<50$ cells $/ \mathrm{mm}^{3}$ ). However, many cohorts from both resource-limited and developed clinical settings report that approximately a third of $\mathrm{HIV}$-infected patients present late (based on $\mathrm{CD}^{+}{ }^{+} \mathrm{T}$-cell count $<200$ cells $/ \mathrm{mm}^{3}$ or the WHO stage 3 or 4) [3-7], with some cohorts reporting dramatically higher rates [8].
In countries where ART is readily available, late presentation is still one of the leading causes of HIV-related mortality in HIV-infected patients [9]. Overall it is estimated that late presenters have over three-times higher mortality rates compared with nonlate presenters over a similar follow-up period [10]. In addition to the consequences for the patient in terms of illness, and society in terms of ongoing transmission of HIV infection, caring for late presenters incurs greater healthcare costs, estimated to be more than twice that of nonlate presenters and not solely explained by direct inpatient care costs [11].

Ideally, this challenging issue should be addressed by diagnosing and treating late presenters well before they advance to symptomatic HIV infection. Strategies to increase HIV testing, including the introduction of opt-out screening programs and targeted point-of-care testing [12] are increasingly being researched but may not necessarily solve the issue of late presenters alone. Middle-aged, male heterosexuals are frequently over-represented in cohort studies of late presenters [7]. Many such patients may have no reason to visit their physician unless they develop clinical symptoms. In addition, being born outside the country of residence and the presence of barriers to healthcare access also frequently rank among factors associated with late presentation $[6,7,13]$. Taking these issues into account, many late presenters may not have access to routine healthcare and, therefore, opportunities to test these individuals through conventional healthcare approaches may be lacking. In addition, patients such as middle-aged males who do not openly admit risk exposure may not be considered at high risk of acquiring HIV infection by their primary physician and, therefore, diagnostic opportunities may also be missed, even when a patient presents with symptoms.

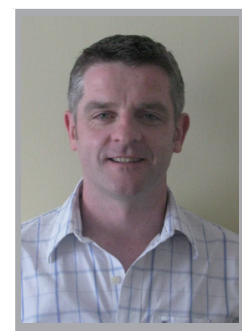

Patrick W Mallon

School of Medicine \& Medical Sciences, University College Dublin, Dublin 4, Ireland and

Department of Infectious Diseases, Mater Misericordiae University Hospital,

Eccles Street, Dublin 7, Ireland

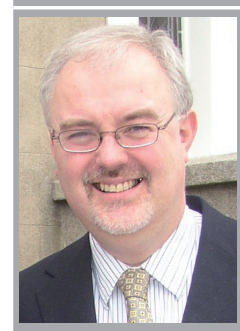

William G Powderly

Author for correspondence:

Dublin Academic Medical

Centre, School of Medicine

\& Medical Sciences,

University College Dublin,

Dublin 4, Ireland

Tel.: +353 I7I6 6609;

Fax: +353 I7I6 6660;

head.smms@ucd.ie 
To address this issue, the identification of a set of AIDS indicator diseases may help increase direct tests at the point of healthcare contact [14]. The most effective strategy would probably be directed to the education of relevant healthcare providers coordinated with an increased public awareness and drive towards reducing stigma through national information campaigns.

In addition to measures aimed at the identification of patients before they reach the stage of late presentation, a better understanding of the management of opportunistic infections in the setting of advanced disease is essential to reduce the morbidity and mortality associated with this clinical presentation. For example, despite the fact that combination ART has been in use for the best part of a decade, until recently, issues such as the optimal timing of ART initiation in patients presenting with AIDS was unclear.

\section{"Ideally, this challenging issue should be addressed by diagnosing and \\ treating late presenters well before they advance to symptomatic HIV infection."}

The recent publication of the AIDS Clinical Trial Group (ACTG) A5164 study sheds light on this issue. This prospective, multicenter, randomized study examined the effects of early versus deferred initiation of combination ART in patients presenting with an opportunistic infection (OI) [15]. Patients in the 'early' category received ART within 14 days of initiation of treatment for an OI, whilst those randomized to the deferred arm received ART once treatment of the OI was complete. Subjects were classified into three outcome categories; best being those without disease progression with a suppressed HIV RNA at week 48, intermediate being those without disease progression but not virally suppressed at week 48 (HIV RNA > 50 copies $/ \mathrm{ml}$ ) and worst being those patients who experienced disease progression or death. The 283 randomized subjects were predominantly male (85\%), 23\% were Caucasian, the median age was 38 years, $70 \%$ had a baseline CD4+ $4^{+}$-cell count below 50 cells $/ \mathrm{mm}^{3}$ and most (89\%) received protease inhibitor-containing ART.

Although there was no statistical difference in the primary outcome between the early and deferred arms, secondary analyses of clinical outcome revealed patients treated early with ART experienced less disease progression and death compared with the deferred arm (OR: 0.51; 95\% CI: 0.27-0.94; p = 0.035) despite similar rates of $\mathrm{CD}^{+}{ }^{+} \mathrm{T}$-cell count recovery (median $=220$ vs 233 cells $/ \mathrm{mm}^{3}$ in the immediate vs deferred arms, respectively, at week 48), virological efficacy of the regimens (slightly more than $70 \%$ in both arms undetectable at week 48 ) and no difference in the overall duration of hospital stay. Although both study arms achieved similar $\mathrm{CD}^{+}{ }^{+} \mathrm{T}$-cell and HIV RNA targets at week 48 , the difference in clinical outcome may be partially explained by a more rapid recovery of $\mathrm{CD}^{+} \mathrm{T}$ cells in those randomized to early ART.

One of the concerns surrounding early ART initiation in the presence of active OIs has been the development of immune reconstitution disease (IRD). However, in the ACTG A5164 study, IRD was observed in only $7 \%$ of subjects, and did not differ between arms. This rate was lower than would have been expected from some previous studies [16,17], although it is consistent with the incidence $(10 \%)$ observed recently in a large prospective study in South Africa [18]. This may partly be explained by a recruitment selection bias towards patients with less severe disease (and, therefore, a more favorable outcome) that is inherent in prospective clinical trials although the fact that the median baseline CD $4^{+}$T-cell count was 29 cells $/ \mathrm{mm}^{3}$ and $70 \%$ patients had a CD4 ${ }^{+}$T-cell count below 50 cells $/ \mathrm{mm}^{3}$ [15] would suggest that these patients were indeed at risk of IRD.

Another potential explanation for lower rates of IRD is that patients co-infected with Mycobacterium tuberculosis (TB) were not recruited to ACTG A5162. This also raises questions as to whether the treatment approach of early ART initiation after presentation with an OI can be extrapolated to patients suffering from TB. A retrospective study of 160 patients with TB and HIV infection initiating ART exhibited symptoms broadly classified as IRD in 19 patients (12\%) after ART initiation, with the highest rates occurring in patients with short intervals between initiation of treatment for TB and initiation of ART ( $\leq 30$ days) [19]. Although most cases were self-limiting and did not require additional therapy, two deaths attributable to this clinical syndrome were recorded, both in patients with disseminated TB.

The effective management of HIV-TB coinfection, a complex but common clinical scenario, will rely on the development of clear definitions to describe the various clinical presentations of TB in patients initiating ART [20,21], coupled with additional research into the best treatment approaches (including 
optimal time for ART initiation) for each specific type of presentation; in particular, defining and differentiating patients suffering from the 'unmasking' of TB upon ART initiation from those experiencing true IRD. Several large trials investigating the use of early ART in HIV/TB are ongoing. The initial results of one such study - Starting ART in Three Points in TB Therapy (SAPIT) trial - were recently presented [22]. This study randomly assigned 645 patients with a CD4 ${ }^{+}$T-cell count below 500 cells $/ \mathrm{mm}^{3}$ and a positive acid-fast bacillus smear for TB to one of three arms - starting ART at TB treatment initiation, starting ART after the intensive phase of TB therapy or starting ART after TB treatment is complete. The results for the first two groups were combined into the 'integrated' group, and compared with the third arm (the 'sequential' group). The trial's Data and Safety Monitoring Board halted the trial when the mortality of patients in the integrated group was $56 \%$ lower than those in the sequential group. Results of a recently published Spanish cohort study [23], in which early use of ART in HIV-positive patients with TB was associated with a $63 \%$ reduction in mortality, also favor early initiation of ART in this setting. Although these two studies support combined ART and TB treatment, the investigation into the optimal time to initiate ART in HIV-TB co-infected patients (concomitant with initiation of anti-TB treatment or after intensive TB therapy) awaits the results of ongoing trials.

In the interim, it is clear for patients presenting with OIs other than TB, the early initiation of ART near the time of starting OI treatment should be considered in most cases, unless there are individual compelling contraindications [101].

\section{Financial \& competing interests disclosure}

Patrick Mallon has received support in the form of honoraria, research grants, lecture sponsorships or advisory board honoraria from BMS, Abbott, Merck Sharp and Dohme, Pfizer, Gilead, GSK, Roche and Boehringer-Ingelheim. William Powderly has received support from BMS, Tibotec and Boehringer-Ingelheim. The authors have no other relevant affiliations or financial involvement with any organization or entity with a financial interest in or financial conflict with the subject matter or materials discussed in the manuscript apart from those disclosed.

No writing assistance was utilized in the production of this manuscript.

\section{Bibliography}

Papers of special note have been highlighted as:

- of interest

" - of considerable interest

1 Palella FJ Jr, Delaney KM, Moorman AC et al.: Declining morbidity and mortality among patients with advanced human immunodeficiency virus infection. HIV Outpatient Study Investigators. N. Engl. J. Med. 338, 853-860 (1998).

2 Marin B, Thiebaut R, Bucher HC et al.: Non-AIDS-defining deaths and immunodeficiency in the era of combination antiretroviral therapy. AIDS 23(13), 1743-1753 (2009).

3 Borghi V, Girardi E, Bellelli S et al.: Late presenters in an HIV surveillance system in Italy during the period 1992-2006. J. Acquir. Immune Defic. Syndr. 49, 282-286 (2008).

4 Wolbers M, Bucher HC, Furrer $\mathrm{H}$ et al.: Delayed diagnosis of HIV infection and late initiation of antiretroviral therapy in the Swiss HIV Cohort Study. HIV Med. 9, 397-405 (2008).

5 Kigozi G, Watya S, Polis CB et al.: The effect of male circumcision on sexual satisfaction and function, results from a randomized trial of male circumcision for human immunodeficiency virus prevention, Rakai, Uganda. BJU Int. 101, 65-70 (2008).

6 Girardi E, Aloisi MS, Arici C et al:: Delayed presentation and late testing for HIV: demographic and behavioral risk factors in a multicenter study in Italy. J. Acquir. Immune Defic. Syndr. 36, 951-959 (2004).

7 Bonjour MA, Montagne M, Zambrano M et al.: Determinants of late disease-stage presentation at diagnosis of HIV infection in Venezuela: a case-case comparison. AIDS Res. Ther. 5, 6 (2008).

8 Jean-Jacques M, Walensky RP, Aaronson WH, Chang Y, Freedberg KA: Late diagnosis of HIV infection at two academic medical centers: 1994-2004. AIDS Care 20, 977-983 (2008).

9 Lucas SB, Curtis H, Johnson MA: National review of deaths among HIV-infected adults. Clin. Med. 8, 250-252 (2008).

10 Delpierre C, Lauwers-Cances V, Pugliese P et al.: Characteristics trends, mortality and morbidity in persons newly diagnosed HIV positive during the last decade: the profile of new HIV diagnosed people. Eur. J. Public Health. 18, 345-347 (2008).

11 Krentz HB, Kliewer G, Gill MJ: Changing mortality rates and causes of death for HIV-infected individuals living in Southern Alberta, Canada from 1984 to 2003. HIV Med. 6, 99-106 (2005).

12 Calderon Y, Leider J, Hailpern S et al.: A randomized control trial evaluating the educational effectiveness of a rapid HIV posttest counseling video. Sex. Transm. Dis. 36, 207-210 (2009).

13 Hocking JS, Rodger AJ, Rhodes DG, Crofts N: Late presentation of HIV infection associated with prolonged survival following AIDS diagnosis - characteristics of individuals. Int. J. STD AIDS 11, 503-508 (2000)

14 Gazzard B, Clumeck N, d'Arminio Monforte A, Lundgren JD: Indicator disease-guided testing for HIV - the next step for Europe? HIV Med. 9(Suppl. 2), 34-40 (2008).

- Identification of indicator illnesses as an attempt to improve rates of HIV testing. 
15 Zolopa A, Andersen J, Powderly W et al.: Early antiretroviral therapy reduces AIDS progression/death in individuals with acute opportunistic infections: a multicenter randomized strategy trial. PLoS ONE 4, e5575 (2009).

- Major randomized trial demonstrating clinical benefit of starting antiretroviral therapy in patients with acute opportunistic infection.

16 Jevtovic DJ, Salemovic D, Ranin J, Pesic I, Zerjav S, Djurkovic-Djakovic O: The prevalence and risk of immune restoration disease in HIV-infected patients treated with highly active antiretroviral therapy. HIV Med. 6, 140-143 (2005).

17 Shelburne SA, Visnegarwala F, Darcourt J et al.: Incidence and risk factors for immune reconstitution inflammatory syndrome during highly active antiretroviral therapy. AIDS 19, 399-406 (2005).
18 Murdoch DM, Venter WD, Feldman C, van Rie A: Incidence and risk factors for the immune reconstitution inflammatory syndrome in HIV patients in South Africa: a prospective study. AIDS 22, 601-610 (2008).

19 Lawn SD, Myer L, Bekker LG, Wood R: Tuberculosis-associated immune reconstitution disease: incidence, risk factors and impact in an antiretroviral treatment service in South Africa. AIDS 21, 335-341 (2007).

20 Lawn SD, Wilkinson RJ, Lipman MC, Wood R: Immune reconstitution and "unmasking" of tuberculosis during antiretroviral therapy. Am. J. Respir. Crit. Care Med. 177, 680-685 (2008).

21 Meintjes G, Lawn SD, Scano F et al.: Tuberculosis-associated immune reconstitution inflammatory syndrome: case definitions for use in resource-limited settings. Lancet Infect. Dis. 8, 516-523 (2008).

Important attempt to clinically define immune reconstitution inflammatory syndrome for research purposes.
22 Abdool Karim S, Naidoo K, Grobler A et al.: Initiating ART during TB treatment significantly increases survival: results of a randomized controlled clinical trial in TB/HIV-co-infected patients in South Africa. Proceedings of the 16th Conference on Retroviruses and Opportunistic Infections. Montreal, Canada, 8-11 February 2009 (Abstract 36a).

23 Velasco M, Castilla V, Sanz J et al.: Effect of simultaneous use of highly active antiretroviral therapy on survival of HIV patients with tuberculosis. J. Acquir. Immune Defic. Syndr. 50, 148-152 (2009).

\section{- Website}

101 Guidelines for prevention and treatment of opportunistic infections among HIV-exposed and HIV-infected children.

2008 recommendations from the NIH, the CDC and the HIV Medicine Association of the Infectious Diseases Society of America (HIVMA/IDSA).

http://adisinfo.nih.gov/contentfiles/Adult_OI. pdf. 